\title{
Male-Produced Aggregation Pheromones of the Cerambycid Beetles Xylotrechus colonus and Sarosesthes fulminans
}

\author{
Emerson S. Lacey • Jocelyn G. Millar • \\ Jardel A. Moreira $\cdot$ Lawrence M. Hanks
}

Received: 20 January 2009/Revised: 20 March 2009 /Accepted: 4 April 2009/Published online: 15 May 2009

(C) The Author(s) 2009. This article is published with open access at Springerlink.com

\begin{abstract}
Adults of both sexes of the cerambycid beetles Xylotrechus colonus (F.) and Sarosesthes fulminans (F.) were attracted to odors produced by male conspecifics in olfactometer bioassays. Analyses of headspace volatiles from adults revealed that male $X$. colonus produced a blend of $(R)$ - and ( $S$ )-3-hydroxyhexan-2-one and $(2 S, 3 S)$ - and $(2 R, 3 R)-2,3$-hexanediol, whereas male $S$. fulminans produced $(R)$-3-hydroxyhexan-2-one and $(2 S, 3 R)$-2,3-hexanediol. All of these compounds were absent in the headspace of females. Two field bioassays were conducted to confirm the biological activity of the synthesized pheromones: (1) enantiomerically enriched pheromone components were tested singly and in species-specific blends and (2) fourcomponent mixture of racemic 3-hydroxyhexan-2-one plus racemic 2-hydroxyhexan-3-one and the four-component blend of the stereoisomers of 2,3-hexanediols were tested separately and as a combined eight-component blend. In these experiments, adult male and female $X$. colonus were captured in greatest numbers in traps baited with the reconstructed blend of components produced by males, although significant numbers were also captured in traps
\end{abstract}

E. S. Lacey $(\bowtie) \cdot$ L. M. Hanks $(\bowtie)$

Department of Entomology,

University of Illinois at Urbana-Champaign,

Urbana, IL 61801, USA

e-mail: eslacey@ucr.edu

e-mail: hanks@life.illinois.edu

J. G. Millar J J. A. Moreira

Department of Entomology, University of California,

Riverside, CA 92521, USA

Present Address:

E. S. Lacey

Department of Entomology, University of California, Riverside, CA 92521, USA baited with $(R)$-3-hydroxyhexan-2-one alone or in blends with other compounds. Too few adult $S$. fulminans were captured for a statistical comparison among treatments, but all were caught in traps baited with lures containing $(R)-3$ hydroxyhexan-2-one. In addition to these two species, adults of two other species of cerambycid beetles, for which pheromones had previously been identified, were caught: Neoclytus a. acuminatus (F.) and its congener Neoclytus m. mucronatus (F.). Cross-attraction of beetles to pheromone blends of other species, and to individual pheromone components that are shared by two or more sympatric species, may facilitate location of larval hosts by species that compete for the same host species.

Keywords Cerambycidae $\cdot$ Wood-borer $\cdot$ Insect communities · Sex pheromone - Aggregation pheromone . (R)-3-hydroxyhexan-2-one

\section{Introduction}

Aggregation or sex pheromones produced by males have been identified for 12 species of longhorned beetles of the subfamily Cerambycinae (Coleoptera: Cerambycidae; Lacey et al. 2004, 2007b; Hanks et al. 2007; Ray et al. 2009a, b, and references therein). Pheromone components of most of these species are typically six to ten carbons long with hydroxyl or carbonyl groups at $\mathrm{C}_{2}$ and $\mathrm{C}_{3}$ (Lacey et al. 2004, 2007b; but see Lacey et al. 2008; Hanks et al. 2007; Ray et al. 2009a, b). In addition, minor components that are different from this general structural motif may be important synergists in the pheromone (Fettköther et al. 1995; Reddy et al. 2005; Lacey et al. 2008).

In this article, we describe investigations of the chemical ecology of two sympatric and synchronic cerambycine 
species of the tribe Clytini, Xylotrechus colonus (F.) and Sarosesthes fulminans (F.). Larvae of both species develop in woody tissues of stressed, moribund, and damaged (often wind-thrown) trees of a variety of hardwood species (especially of the genera Caryae and Quercus; for general biology, see Linsley 1964). Both species are native to eastern North America. Adults are active in spring and summer and are crepuscular, flying from $\sim 16: 00$ to $22: 00 \mathrm{~h}$ (ESL, personal observation). Adult male and female $X$. colonus commonly aggregate, in numbers that may exceed 30 individuals per group, on larval hosts. Adult $S$. fulminans may be present on the same larval hosts, but in much smaller numbers (ESL, personal observation). Adult male $X$. colonus and $S$. fulminans have pores on the surface of the prothorax that are characteristic of pheromone production in other species of cerambycines (Ray et al. 2006). Male $X$. colonus sometimes also display a characteristic posture that has been associated with pheromone release in other cerambycine species (the "push-up stance"; Lacey et al. 2007a).

We tested the hypotheses that male $X$. colonus and $S$. fulminans produce aggregation pheromones that conform to the structural motif of other cerambycines and that complete blends of synthetic pheromone components are more attractive to beetles than individual components. We also tested the responses of both species to traps baited with blends of all regio- and stereoisomers of the main pheromone components. These blends are more economical to synthesize than enantiomerically enriched pheromone components and have proven effective as attractants for other cerambycine species (Hanks et al. 2007).

\section{Methods and Materials}

Source of Insects Adult X. colonus and S. fulminans were collected by hand on 6 June 2004 from a wind-thrown white oak, Quercus alba L., at Allerton Park (Piatt Co., IL, USA), a 600-ha mixed hardwood forest owned by the University of Illinois. Adults were housed individually in the laboratory $\left(12: 12 \mathrm{~h} \mathrm{~L}: \mathrm{D}, \sim 20^{\circ} \mathrm{C}, \sim 50 \% \mathrm{RH}\right)$ in cylindrical cages, constructed of aluminum window screen, with plastic Petri dishes at top and bottom, and provided with $10 \%$ sucrose in water. Beetles used in bioassays were active and apparently healthy.

Olfactometer Bioassays We tested for volatile attractants in both species by using a horizontal glass Y-tube olfactometer (6 $\mathrm{cm}$ diameter, main tube $26 \mathrm{~cm}$ long, arm length $22 \mathrm{~cm}$, and $70^{\circ}$ angle between arms). We conducted olfactometer studies outdoors in partial shade because all cerambycine species that we have bioassayed to date did not respond in Y-tube olfactometer bioassays conducted under laboratory conditions (see Lacey et al. 2004, 2007b, 2008). A 2-L plastic chamber, containing a cylinder of aluminum screen for a perch, was attached to each arm of the Y-tube. When bioassaying $X$. colonus, one chamber held six males and the other held six females. Only three S. fulminans of each sex were used as odor sources in their respective bioassay, because this species was less abundant. Ambient air was pulled through the olfactometer (air speed $2.5 \mathrm{~m} \mathrm{~s}^{-1}$ ) with a $0.75 \mathrm{~kW}$ vacuum cleaner connected to a variable voltage power supply. For each trial, a beetle was released at the base of the Y-tube and allowed $10 \mathrm{~min}$ to respond (crossing a line $18 \mathrm{~cm}$ down one arm) to an odor source. Chambers were alternated between Y-tube arms every three trials to control for positional bias. The chambers and olfactometer were washed with unscented laboratory detergent (Alconox ${ }^{\circledR}$ powder, Alconox, Inc., NY, USA) and rinsed with acetone each day. We bioassayed $20 \mathrm{X}$. colonus of each sex between 17:00-20:00 h on 9, 10, and 13 June 2004 and 13 female and seven male $S$. fulminans during the same hours on 11 and 12 June 2004 (skies clear, air temperatures 24$30^{\circ} \mathrm{C}$ ). For each species, numbers of each sex responding to treatments were compared with the $\chi^{2}$ goodness-of-fit test corrected for continuity (Sokal and Rohlf 1995).

Identification of Pheromone Components Volatile compounds produced by adult $X$. colonus and S. fulminans were collected by placing five adult females and males in separate glass vacuum traps $(0.3 \mathrm{~L}$, manufactured by the glass shop, School of Chemical Sciences, UIUC) that were lined with aluminum screen to provide perches. A glass tube $(6 \mathrm{~cm}$ long $\times 9.5$ o.d. $\times 4 \mathrm{~mm}$ i.d. $)$ containing $100 \mathrm{mg}$ of 80/100 mesh SuperQ ${ }^{\circledR}$ (Alltech Associates, Deerfield, IL, USA) was attached to one nipple of each chamber with an 8-cm long section of Teflon ${ }^{\circledR}$ tubing. Charcoal-purified air was pulled through the apparatus with a water aspirator $\left(1 \mathrm{~L} \mathrm{~min}^{-1}\right)$. Males and females of each species were aerated simultaneously on a laboratory windowsill from 16:00 to 22:00 h: X. colonus on 11, 12, and 13 June and $S$. fulminans on 14, 15, and 16 June 2004. We selected these time periods for collecting volatiles from beetles because they corresponded to activity periods in the field (ESL, personal observation). Collectors were eluted into silanized glass vials (Cat. \#27114, Supelco ${ }^{\circledR}$, Bellefonte, PA, USA) with three $0.5-\mathrm{ml}$ aliquots of methylene chloride. The resulting extracts were analyzed with a Hewlett-Packard ${ }^{\circledR}$ (Sunnyvale, CA, USA) 6890 gas chromatograph (GC) coupled to a 5973 mass selective detector (MS). The GC was fitted with a DB5-MS column $(30 \mathrm{~m} \times 0.25 \mathrm{~mm}, 25 \mu \mathrm{m}$ film thickness; Agilent Technologies, Santa Clara, CA, USA) and programmed from $40^{\circ} \mathrm{C}$ (held for $1 \mathrm{~min}$ ) to $250^{\circ} \mathrm{C}$ at $10^{\circ} \mathrm{C} \mathrm{m^{-1 }}$ (held for $15 \mathrm{~min}$ ), with an injector temperature of $250^{\circ} \mathrm{C}$. Injections were made in the splitless mode. When it became clear that one or more of the 
compounds in the extract might be thermally unstable (see Leal et al. 1995; Lacey et al. 2007b), the injector temperature was lowered to $100^{\circ} \mathrm{C}$, and the temperature program was changed to $20^{\circ} \mathrm{C}$ (held for $2 \mathrm{~min}$ ) to $250^{\circ} \mathrm{C}$ at $10^{\circ} \mathrm{C} \mathrm{min}^{-1}$ (held for $15 \mathrm{~min}$ ). Absolute configurations of the insect-produced compounds were determined by analysis of aliquots on a Cyclodex-B GC column (30 m× $0.25 \mathrm{~mm}, 0.25$ micron film thickness, J\&W Scientific, Folsom CA, USA) with the GC programmed from $50^{\circ} \mathrm{C}$ (held for $1 \mathrm{~min}$ ) to $200^{\circ} \mathrm{C}$ at $5^{\circ} \mathrm{C} \mathrm{min}{ }^{-1}$, injector $100^{\circ} \mathrm{C}$, detector $200^{\circ} \mathrm{C}$. Identifications of peaks were confirmed by coinjections of extracts with authentic standards.

Field Bioassays of Synthetic Pheromone In Experiment 1, bioassays of reconstructed blends of male-specific volatiles for each of the two species, as well as of the individual components, were conducted on 9 days between 11 June and 27 July 2006 and 5 days between 29 May and 14 June 2007. Bioassays were carried out at Allerton Park and Brownfield Woods (Champaign Co., IL; skies clear to partly cloudy, maximum air temperatures $\sim 22-30^{\circ} \mathrm{C}$, wind speed $\sim 8-20 \mathrm{kph}$ ), the latter a 26-ha mixed deciduous forest owned by the University of Illinois. We used two different sites because we observed that trap catches of cerambycine species at individual sites decline over time, possibly because trapping depletes local populations (ESL, unpublished data). Both of the sites had populations of $X$. colonus and S. fulminans (ESL, personal observation) and replications of the experiment were assigned randomly to sites.

Traps were black cross-vane flight intercept panel traps (1.2 $\mathrm{m}$ tall $\times 0.30 \mathrm{~m}$ wide, Intercept $\mathrm{t}^{\mathrm{TM}}$, model PT, APTIV, Inc., Portland, OR, USA). Traps were suspended from hangers constructed of $1.27 \mathrm{~cm}$ i.d. PVC pipe (D1785, Schedule 40, Charlotte Pipe and Foundry Co., Charlotte, $\mathrm{NC}$, USA), with a $1.5 \mathrm{~m}$ long upright connected with a $\mathrm{T}$ fitting to a $20-\mathrm{cm}$ long arm with a loop of wire at the end to which the trap was attached. The upright was mounted on a $1.5 \mathrm{~m}$ section of $1.27 \mathrm{~cm}$ diameter steel reinforcing bar driven into the ground. Traps were positioned $10 \mathrm{~m}$ apart in a straight line approximately perpendicular to the prevailing wind direction. Traps were set up at $\sim 14: 00 \mathrm{~h}$ and beetles were removed from traps at $\sim 11: 00 \mathrm{~h}$ the following day.

Enantiomerically enriched hydroxyhexanones (94\% ee; Lacey et al. 2007a, b) and hexanediols ( $>98 \%$ stereoisomerically pure, Lacey et al. 2008) were synthesized as previously described. Each treatment was dissolved in $1 \mathrm{ml}$ of absolute ethanol. We randomly assigned each of the following nine treatments to traps: (1) $X$. colonus blend (see "Results"): (R)-3-hydroxyhexan-2-one (8 mg), (S)-3hydroxyhexan-2-one $(1.25 \mathrm{mg}),(2 R, 3 R)-2,3$-hexanediol (2.5 mg), and (2S,3S)-2,3-hexanediol (0.5 mg); (2) $S$. fulminans blend (see "Results"): $(R)$-3-hydroxyhexan-2-one
(8 mg) and $(2 S, 3 R)-2,3$-hexanediol (1.25 mg); (3) (R)-3hydroxyhexan-2-one ( $8 \mathrm{mg})$; (4) $(S)$-3-hydroxyhexan-2-one (8 mg); (5) (2S,3 S)-2,3-hexanediol (8 mg); (6) (2R,3R)-2,3hexanediol ( $8 \mathrm{mg})$; (7) $(2 S, 3 R)$-2,3-hexanediol (8 mg); (8) ethanol control $(1 \mathrm{ml})$; and (9) blank control (empty emitter).

Pheromones were loaded into release devices that consisted of modified 1.5-ml microcentrifuge tubes (\#05406-16; Fisher Scientific, Pittsburg, PA, USA). A section of polyethylene tubing ( $25 \mathrm{~mm}$ long, $4 \mathrm{~mm}$ o.d., $2 \mathrm{~mm}$ i.d.) was inserted through a $4 \mathrm{~mm}$ hole drilled through the tube cap and cemented in place with quick setting epoxy glue such that $8 \mathrm{~mm}$ of tubing extended beyond the cap. A $5-\mathrm{cm}$ long section of pipe cleaner (cotton fiber and steel wire, $2.5 \mathrm{~mm}$ diameter) was inserted through the tubing such that one end reached the bottom of the microcentrifuge tube and the other end extended $5 \mathrm{~mm}$ beyond the end of the polyethylene tubing (modified from Hanks et al. 2007). Ethanol is an efficient carrier of hydroxyhexanones and hexanediols (Hanks et al. 2007) and the lures released $\sim 1 \mathrm{ml}$ of ethanol solution over the course of 1 day under field conditions (unpublished data). Lures were hung with wire in the open central slot of panel traps.

Differences among treatments in numbers of beetles captured (sexes combined) were tested with the nonparametric Friedman's test (blocked by site and day; PROC FREQ with CMH option, SAS Institute, 2001) because assumptions of analysis of variance were violated by heteroscedasticity (Sokal and Rohlf 1995). Differences between pairs of means were tested with the REGWQ means-separation test to control maximum experiment-wise error rates (SAS Institute, 2001). Replications that captured fewer than two beetles overall $(N=9)$ were excluded from the statistical analyses for a given species. Differences between numbers of females and males captured within treatments were tested with the $\chi^{2}$ goodness-of-fit test (Sokal and Rohlf 1995).

We conducted a further trial ('Experiment 2') to test the responses of $X$. colonus and $S$. fulminans to blends of stereoisomers of the chemicals to determine whether unnatural regio- and/or stereoisomers had an inhibitory effect. This bioassay was conducted on 25 days between 13 June-31 August 2006 and 18 days between 5 May-15 August 2007 at Allerton Park and Brownfield Woods using the methods described above. Treatments were as follows (compounds synthesized as described in Hanks et al. (2007) and diluted to $1 \mathrm{ml}$ with absolute ethanol): (1) $\sim 1: 1: 1: 1$ blend of enantiomers of 3-hydroxyhexan-2-one and 2hydroxyhexan-3-one (100 mg total load, $25 \mathrm{mg}$ of each isomer; henceforward referred to as "generic hydroxyhexanones"); (2) 1:1:1:1 blend of all four stereoisomers of 2,3-hexanediol (100 mg total load, $25 \mathrm{mg}$ of each isomer; henceforward "generic hexanediols"); (3) 1:1 combination 
of generic hydroxyhexanones and generic hexanediols (200 mg total load); (4) ethanol control (1 ml); and (5) blank control (empty emitter).

The mixtures were loaded into lures and differences among treatments in numbers of beetles captured per species were tested as in the previous bioassay. Replications that captured fewer than two beetles of a given species were excluded from statistical analysis $(N=28)$. On days when enantiomerically enriched pheromones and generic pheromones were bioassayed simultaneously, trap lines were separated by $>1 \mathrm{~km}$.

\section{Results}

Olfactometer Bioassays In olfactometer bioassays, 17 female $X$. colonus were attracted to odors emitted by live males, compared to only two that responded to odors of live females $\left(\chi^{2}=11.8, P<0.001\right)$. Male $X$. colonus showed a similar response, with 16 responding to odors of live males compared to only one responding to odors from live females $\left(\chi^{2}=13.2\right.$; $P<0.001)$. Both sexes of $S$. fulminans were attracted to odors produced by male conspecifics, with 12 females responding to males and only one responding to females $\left(\chi^{2}=9.31 ; P<\right.$ $0.01)$ and all seven males responding to males $\left(\chi^{2}=7.00 ; P<\right.$ $0.05)$. These findings confirmed that males of both species produce volatile pheromones that attract both sexes.

Identification of Pheromone Components GC-MS analyses of volatiles collected from male $X$. colonus revealed two major peaks in the total ion chromatogram that were absent in the analogous headspace of females. The retention time and mass spectrum of the larger peak (base peak at $m / z 55$, other significant fragments at $m / z 43$ [78\%], 73 [53\%], and 45 [26\%]) matched those of a synthetic standard of 3hydroxyhexan-2-one. The retention time and mass spectrum of the smaller peak (base peak at $\mathrm{m} / \mathrm{z} 55$, other significant fragments at $m / z 75$ [10\%], 73 [61\%], 72 [34\%], $45[26 \%]$, and $43[35 \%])$ matched those of a synthetic standard of 2,3-hexanediol. The stereoisomeric compositions of the insect-produced compounds were determined to be $\sim 70 \%(R)$ - and 10\% $(S)$-3-hydroxyhexan-2-one and $17 \%$ $(2 S, 3 S)$ - and $3 \%(2 R, 3 R)-2,3$-hexanediol by analysis on the Cyclodex-B column, with the enantiomers being resolved to baseline (for elution order of all hydroxyketones and 2,3hexanediols, see Ray et al. 2009b). Analogous collection and analysis of headspace volatiles from both sexes of $S$. fulminans revealed that males produced $\sim 83 \%(R)-3-$ hydroxyhexan-2-one and 17\% (2S,3R)-2,3-hexanediol as the only detectable male-specific components (identified as described above).

Field Bioassays of Synthetic Pheromone In Experiment 1, testing chiral synthetic pheromone components, traps captured 79 adult $X$. colonus of both sexes, but only ten adult $S$. fulminans (Table 1). Treatments differed significantly in numbers of $X$. colonus captured (Fig. 1; Friedman's $\left.Q_{8,79}=52.3, P<0.001\right)$. Only traps baited with $(R)-3$ hydroxyhexan-2-one alone or in a blend (i.e., the $X$. colonus and S. fulminans blends) were significantly attractive compared to ethanol and blank controls (Fig. 1). The blend

Table 1 Results of experiment 1

\begin{tabular}{|c|c|c|c|c|c|c|}
\hline \multirow[b]{2}{*}{ Treatment } & \multicolumn{2}{|c|}{ Xylotrechus colonus } & \multicolumn{2}{|c|}{ Sarosesthes fulminans } & \multicolumn{2}{|c|}{ Neoclytus a. acuminatus } \\
\hline & 우 & $\hat{\sigma}$ & 우 & $\widehat{0}$ & 우 & $\sigma^{\lambda}$ \\
\hline (1) $X$. colonus blend & 17 & 13 & 2 & 1 & 4 & 3 \\
\hline (2) S. fulminans blend & 9 & 9 & 3 & 2 & & \\
\hline (3) (R)-3-hydroxyhexan-2-one & 10 & 7 & 2 & & & \\
\hline (4) (S)-3-hydroxyhexan-2-one & 5 & 3 & & & & \\
\hline (5) $(R, R)$-2,3-hexanediol & 1 & & & & & \\
\hline (6) $(S, R)$-2,3-hexanediol & & & & & 1 & 1 \\
\hline (7) $(S, S)$-2,3,-hexanediol & 3 & & & & 22 & 14 \\
\hline (8) Ethanol control & 1 & 1 & & & & \\
\hline \multicolumn{7}{|l|}{ (9) Blank control } \\
\hline Total & 46 & 33 & 7 & 3 & 27 & 18 \\
\hline
\end{tabular}

Numbers of cerambycid beetles of three species caught in traps baited with enantiomerically enriched candidate pheromone components $(N=9$ replicates for Xylotrechus colonus and Sarosesthes fulminans; $N=10$ replicates for $N$. a. acuminatus). The blend of components specific to $X$. colonus contained $(R)$ - and $(S)$-3-hydroxyhexan-2-one and $(2 R, 3 R)$ - and $(2 S, 3 S)$-2,3-hexanediol. The $S$. fulminans blend contained $(R)-3$ hydroxyhexan-2-one and $(2 S, 3 R)-2,3$-hexanediol. Treatment numbers correspond to treatments in Fig. 1. Sex ratio of total numbers within species were not significantly different from $1: 1\left(\chi^{2}, P>0.05\right)$. 


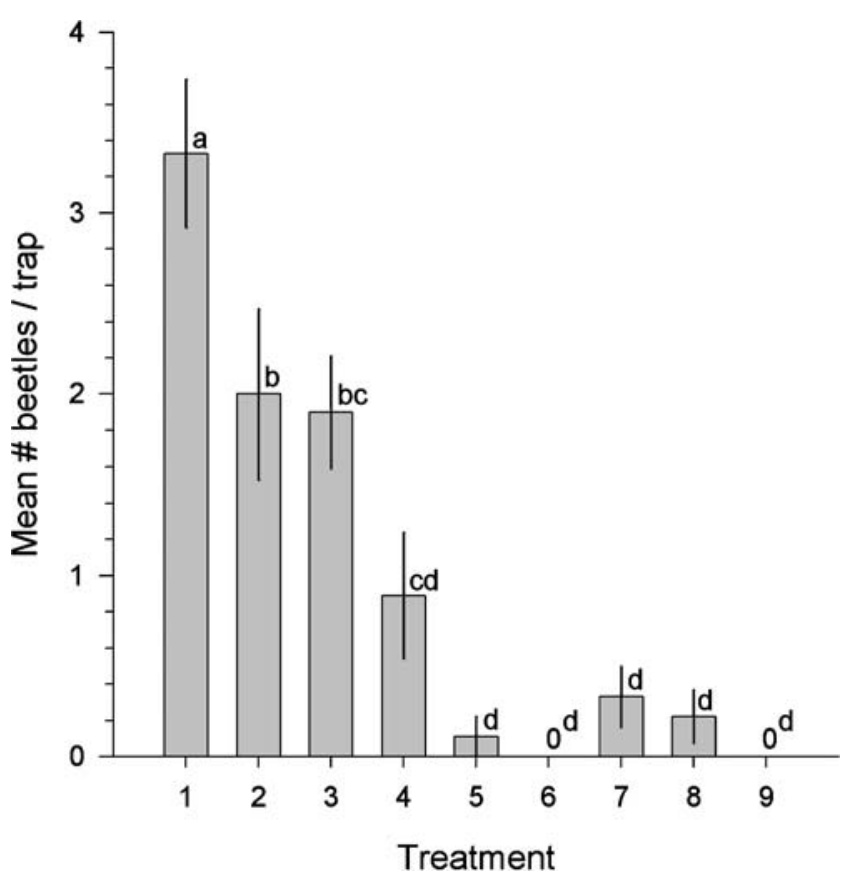

Fig. 1 Mean $( \pm \mathrm{SE})$ numbers of adult Xylotrechus colonus $(N=9$ trials) captured per trap (sexes combined) with respect to composition of the lure ( $N=9$ trials). The blend of components specific for $X$. colonus contained $(R)$ - and $(S)$-3-hydroxyhexan-2-one with $(2 R, 3 R)$ and $(2 S, 3 S)-2,3$-hexanediol, the Sarosesthes fulminans blend contained (R)-3-hydroxyhexan-2-one and (2S,3R)-2,3-hexanediol. Treatment numbers correspond to treatments in Table 1: (1) $X$. colonus blend, (2) S. fulminans blend, (3) $(R)$-3-hydroxyhexan-2-one, (4) (S)-3-hydroxyhexan-2-one, (5) (2R,3R)-2,3-hexanediol, (6) (2 $S, 3 R)$-2,3-hexanediol, (7) (2S,3S)-2,3-hexanediol, (8) ethanol control, and (9) blank control. Means with different letters are significantly different (REGWQ test; $P<0.05$ )

of pheromone components specific for $X$. colonus captured the greatest number of beetles (Fig. 1). Too few adult $S$. fulminans were captured for statistical analysis of treatments; however, all ten beetles were captured with lures that contained the most abundant component of its volatile collection, $(R)$-3-hydroxyhexan-2-one (Table 1).

During Experiment 1, we also trapped adults of another cerambycine species, Neoclytus acuminatus acuminatus (F.) (Table 1), the male-produced pheromone of which is composed solely of ( $2 S, 3 S)$-2,3-hexanediol (Lacey et al. 2004). Treatments differed in numbers of $N$. a. acuminatus captured (Friedman's $Q_{8,45}=64.2, P<0.001$, ten replicates), with only traps baited with $(2 S, 3 S)$-2,3-hexanediol catching significantly more beetles $(3.60 \pm 0.54$, sexes combined) than ethanol and blank controls (means $=0$ for both).

In Experiment 2, testing the generic blends of pheromone stereoisomers, we captured 111 adult $X$. colonus of both sexes (Table 2 ) but only eight $S$. fulminans. Treatments again differed in numbers of adult $X$. colonus captured (Fig. 2, top; Friedman's $Q_{4,111}=70.98, P<0.001$ ), with significantly greater numbers in traps baited with generic hydroxyketones, alone or in combination with generic hexanediols, than in solvent or blank controls. No adult $X$. colonus were captured in traps baited with only the generic hexanediols. Again, too few adult S. fulminans were captured for statistical analysis, although all were captured in traps with lures that contained generic hydroxyketones (Table 2).

In Experiment 2, traps baited with generic pheromone lures also captured 121 adult $N$. a. acuminatus of both sexes, as well as 50 adult males and females of a congener, Neoclytus m. mucronatus (F.) (Table 2). The male-produced pheromone of N. m. mucronatus is composed of only $(R)-3-$ hydroxyhexan-2-one (Lacey et al. 2007b). Treatments differed in numbers of $N$. a. acuminatus and $N . m$. mucronatus captured (Fig. 2; Friedman's $Q_{4,121}=89.3, P<$ $0.001 ; Q_{4,50}=36.3 P<0.001$, respectively). Significantly more $N$. a. acuminatus were captured in traps baited with generic hexanediols, alone or in combination with hydrox-

Table 2 Results of experiment 2

\begin{tabular}{|c|c|c|c|c|c|c|c|c|}
\hline \multirow[b]{2}{*}{ Treatment } & \multicolumn{2}{|c|}{ Xylotrechus colonus } & \multicolumn{2}{|c|}{ Sarosesthes fulminans } & \multicolumn{2}{|c|}{ Neoclytus a. acuminatus } & \multicolumn{2}{|c|}{ Neoclytus m. mucronatus } \\
\hline & q & $\hat{\sigma}$ & q & $\hat{\sigma}$ & 우 & $\hat{\sigma}$ & q & $\hat{\sigma}$ \\
\hline (1) Hydroxyhexanones & 29 & 30 & 3 & 2 & & & 22 & 13 \\
\hline (2) Hexanediols & & & & & 27 & 38 & & 1 \\
\hline (3) Ketones + Diols & 23 & 24 & 2 & 1 & 22 & 32 & 9 & 5 \\
\hline (4) Ethanol control & 2 & 3 & & & & 2 & & \\
\hline \multicolumn{9}{|l|}{ (5) Blank control } \\
\hline Total & 54 & 57 & 5 & 3 & 49 & 72 & 31 & $19^{*}$ \\
\hline
\end{tabular}

Numbers of cerambycid beetles of four species caught in traps baited with generic pheromone blends $(N=31,28,28$, and 18 replicates for Xylotrechus colonus, Sarosesthes fulminans, N. a. acuminatus, and N. m. mucronatus, respectively). The blend of hydroxyhexanones contained equal amounts of $(R)$ - and $(S)$-3-hydroxyhexan-2-one and $(R)$ - and $(S)$-2-hydroxyhexan-3-one, and the blend of hexanediols contained approximately equal amounts of $(2 R, 3 R)-(2 S, 3 S)$ - $(2 R, 3 S)$ - and $(2 S, 3 R)-2,3$-hexanediol. The treatment designated 'Ketones + Diols' contained equal amounts of the blends of hydroxyhexanones and hexanediols. Treatments are numbered to correspond to treatments in Fig. 2.

$* P<0.05\left(\chi^{2}\right)$ next to total number of males indicates sex ratio of beetles captured within species that is significantly different from $1: 1$ 
Fig. 2 Mean $( \pm \mathrm{SE})$ numbers of adults (sexes combined) of three species of cerambycid beetles captured with respect to composition of the lure: top, Xylotrechus colonus ( $N=31$ trials); middle, Neoclytus a. acuminatus ( $N=28$ trials); and bottom, Neoclytus m. mucronatus $(N=$ 18 trials). Treatment numbers correspond to treatments in Table 2: (1) generic hydroxyhexanones; (2) generic hexanediols; (3) 1:1 mixture of generic hydroxyhexanones and hexanediols; (4) ethanol control; and (5) blank control. Means with different letters are significantly different (REGWQ test; $P<0.05$ )

yhexanones (Fig. 2, middle), than in control traps or traps baited with generic hydroxyketones. Conversely, adult $N . m$. mucronatus were only significantly caught in traps baited with generic hydroxyhexanones (Fig. 2, bottom).

In both field bioassays, traps baited with the synthetic pheromone components also captured small numbers (total catch $<8$ individuals) of an additional 11 species of cerambycids, including seven species in the subfamily Cerambycinae (Anelaphus pumilus [Newman], Cyrtophorus verrucosus [Olivier], Knulliana cincta [Drury], Neoclytus caprea [Say], Neoclytus scutellaris [Olivier], Phymatodes amoenus [Say], Xylotrechus convergens LeConte), three species of the subfamily Lamiinae (Liopinus alpha [Say], Psenocerus supernotatus [Say], and Urographis fasciatus [Degeer]), and one species of subfamily Parandrinae (Neandra brunnea [F.]). Traps captured few insects other than the species reported here.

\section{Discussion}

Attraction of both sexes of $X$. colonus and S. fulminans to odors from conspecific males in olfactometer bioassays provided the first evidence that males of both species produce aggregation pheromones. Traps baited with synthetic pheromone captured similar numbers of male and female $X$. colonus, confirming that males produce an aggregation pheromone (Tables 1 and 2). The small number of S. fulminans captured is consistent with its scarcity in the habitats where we conducted our bioassays (ESL, personal observation). The structure of the pheromone components of $X$. colonus and the putative pheromone compounds of $S$. fulminans are consistent with the ketone/diol structural motif of other cerambycine species (see "Introduction") and provide further support for the hypothesis that this structural motif has been conserved within the Cerambycinae (see Lacey et al. 2004, Hanks et al. 2007). Nevertheless, recent studies have shown that some cerambycine species have pheromone components of entirely different chemical classes (Hanks et al. 2007; Lacey et al. 2008; Ray et al. 2009a). Our study also provides further evidence that the presence of gland pores on the prothorax of males (in $X$. colonus, S. fulminans, N. a. acuminatus, and N. m. mucronatus; Ray et al. 2006, Lacey et al. 2007b) and
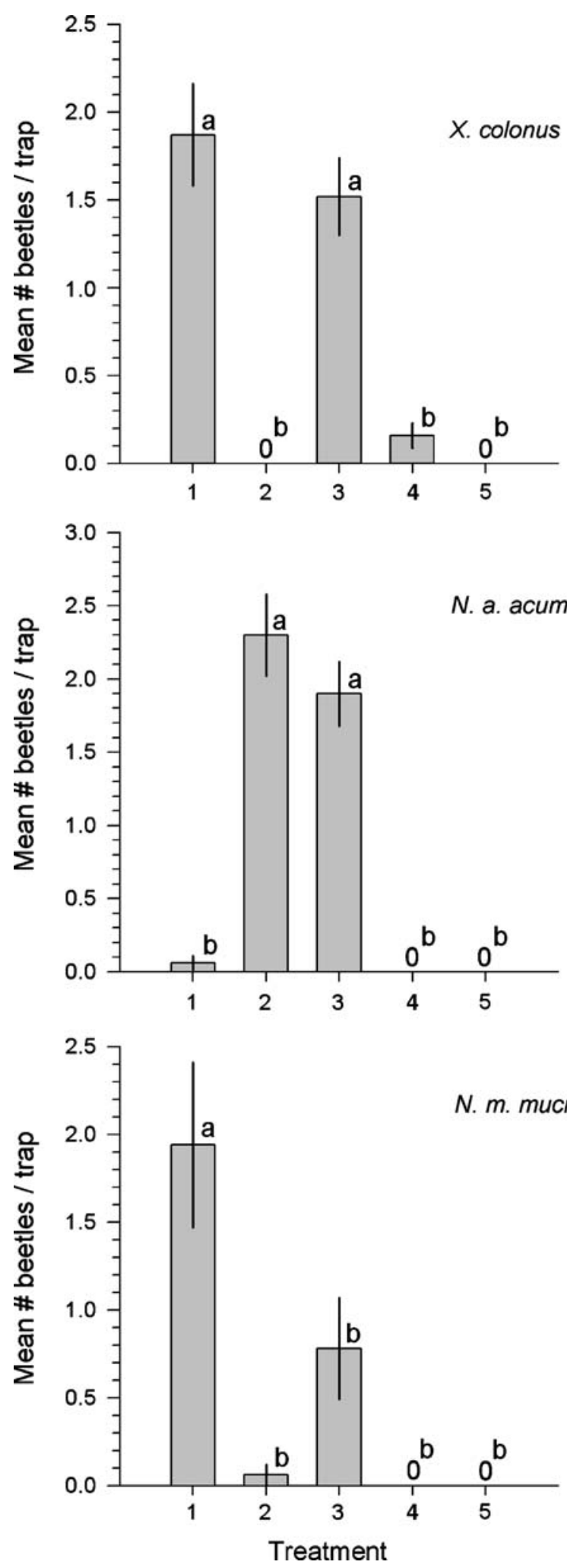

calling behavior of males (in X. colonus, $N$. a. acuminatus, and N. m. mucronatus; Lacey et al. 2007a, b) reliably indicate that volatile pheromones play a role in mate location in cerambycine species. 
In both field experiments, all treatments that contained $(R)$-3-hydroxyhexan-2-one attracted significant numbers of adult $X$. colonus (Figs. 1 and 2 (top)). However, X. colonus were caught in greatest numbers in traps baited with its species-specific blend (Fig. 1), suggesting that the full blend is necessary for optimal attraction. In the absence of (R)-3-hydroxyhexan-2-one, adult $X$. colonus responded weakly, or did not respond (Fig. 1) to the minor components of the blend produced by males [including $(S)$-3-hydroxyhexan-2-one and $(2 R, 3 R)$ - and $(2 S, 3 S)-2,3-$ hexanediols]. Unnatural isomers apparently did not inhibit attraction of $X$. colonus to $(R)$-3-hydroxyhexan-2-one, as evidenced by the significant number of beetles captured in traps baited with the $S$. fulminans lure or the generic hydroxyketones (Figs. 1 and 2 (top)). Attraction of adult males and females of a congener, Xylotrechus nauticus (Mannerheim), to synthetic pheromones also is not inhibited by unnatural isomers (Hanks et al. 2007).

$(R)$-3-Hydroxyhexan-2-one now has been shown to be an important component of male-produced pheromones of 14 species of cerambycine beetles (including $X$. colonus and possibly S. fulminans; see Hanks et al. 2007). Overlap in pheromone composition among sympatric cerambycine species can result in cross-attraction (Hanks et al. 2007). Cross-attraction was evident in the present study, with both sexes of $X$. colonus caught in traps baited with treatments representative of reconstructed blends of the pheromone produced by $S$. fulminans (Fig. 1), as well as the other species caught in traps baited with compounds identified in the two focal species of this study. $X$. colonus, S. fulminans, $N$. a. acuminatus, and $N$. m. mucronatus are sympatric, overlap in phenology, and share larval hosts (Linsley 1964). In fact, adults of all four species may aggregate on the same downed host tree (ESL, personal observation) and adult $X$. colonus and N. m. mucronatus, at least, are attracted to odors emanating from dying hosts (Ginzel and Hanks 2005). Larvae of all four species require hosts that are severely weakened or moribund (Linsley 1964) and competition for these resources (with other insect species and saprophytes), results in rapid degradation of hosts (Hanks 1999). Species that rely on such ephemeral hosts may be under selective pressure to locate hosts quickly and oviposit (Hanks 1999). Thus, these species may exploit the pheromones of other species in their feeding guild as kairomones. Closely related species of bark beetles (Curculionidae: Scolytinae), as well as closely related species of sap beetles (Nitidulidae), share pheromone components, respectively, resulting in cross-attraction among species (e. g., Zilkowski and Bartelt 1999; Haberkern and Raffa 2003). Perhaps not coincidentally, males of these bark and sap beetle species also produce aggregation pheromones (Zilkowski and Bartelt 1999; Haberkern and Raffa 2003) such as those produced by male cerambycine beetles.
The responses of beetles of three species, $X$. colonus, $N$. a. acuminatus, and N. m. mucronatus, to generic lures (Table 2, Fig. 2) again demonstrates that these blends could be used to collect live specimens of various cerambycine species, for analysis and identification of the pheromone blends that they actually produce. In this context, even weak attraction to generic blends may be sufficient for collecting adults for pheromone identification or even monitoring the distribution and phenology of economically important species of interest for which a pheromone has yet to be identified.

Acknowledgements We thank Steve Buck of the UIUC Committee of Natural Areas for access to field sites. This project was supported by the Alphawood Foundation (to LMH), the Exotic/Invasive Pests and Diseases Research Program, University of California, under USDA-CSREES Grant No. 2004-34439-14691 (to JGM and LMH), and the National Research Initiative of the USDA Cooperative State Research, Education and Extension Service, grant number \#200635302-17457 (to LMH and JGM). This research was in partial fulfillment of a PhD degree for ESL from UIUC.

Open Access This article is distributed under the terms of the Creative Commons Attribution Noncommercial License which permits any noncommercial use, distribution, and reproduction in any medium, provided the original author(s) and source are credited.

\section{References}

FettKöther, R., Dettner, K., Schröder, F., Meyer, H., Francke, W., and NOLDT, U. 1995. The male pheromone of the old house borer Hylotrupes bajulus (L.) (Coleoptera: Cerambycidae): identification and female response. Experientia 51:270-277.

GINZEL, M. D., and HANKS, L. M. 2005. Role of host plant volatiles in mate location for three species of longhorned beetles. J. Chem. Ecol. 31:213-217.

HABERKERN, K. E., and RAFFA, K. F. 2003. Phloeophagous and predaceous insects responding to synthetic pheromones of bark beetles inhabiting white spruce stands in the Great Lakes region. J. Chem. Ecol. 29:1651-1663.

HANKS, L. M. 1999. Influence of the larval host plant on reproductive strategies of cerambycid beetles. Annu. Rev. Entomol. 44:483505.

Hanks, L. M., Millar, J. G., Moreira, J. A., Barbour, J. D., LACEy, E. S., MCELFRESh, J. S., REUTER, F. R., and RAY, A. M. 2007. Using generic pheromone lures to expedite identification of aggregation pheromones for the cerambycid beetles Xylotrechus nauticus, Phymatodes lecontei, and Neoclytus modestus modestus. J. Chem. Ecol. 33:889-907.

Lacey, E. S., GinZel, M. D., Millar, J. G., and Hanks, L. M. 2004. Male-produced aggregation pheromone of the cerambycid beetle Neoclytus acuminatus acuminatus. J. Chem. Ecol. 30:14931507.

LACEY, E. S., RAY, A. M., and HANKS, L. M. 2007a. Calling behavior of the cerambycid beetle Neoclytus acuminatus acuminatus. J. Insect Behav. 20:117-128.

Lacey, E. S., Moreira, J. A., Millar, J. G., Ray, A. M., and HanKS, L. M. 2007b. Male-produced aggregation pheromone of the longhorned beetle Neoclytus mucronatus mucronatus. Entomol. Exp. Appl. 122:171-179. 
Lacey, E. S., Moreira, J. A., Millar, J. G., and Hanks, L. M. 2008. A male-produced aggregation pheromone blend consisting of alkanediols, terpenoids, and an aromatic alcohol from the cerambycid beetle Megacyllene caryae. J. Chem. Ecol. 34:408-417.

Leal, W. S., Shi, X., Nakamuta, K., Ono, M., and Meinwald, J. 1995. Structure, stereochemistry, and thermal isomerization of the male sex pheromone of the longhorn beetle Anaglyptus subfasciatus. Proc. Natl. Acad. Sci. U S A 92:1038-1042.

Linsley, E. G. 1964. The Cerambycidae of North America, Part V: Taxonomy and classification of the subfamily Cerambycinae, Tribes Callichromini through Ancylocerini. Univ. Calif. Publ. Entomol. 22:1-197.

Ray, A. M., LaceY, E. S., and Hanks, L. M. 2006. Predicted taxonomic patterns in pheromone production by longhorned beetles. Naturwissenschaften 93:543-550.

Ray, A. M., Millar, J. G., McElfresh, J. S., Swift, I. P., Barbour, J. D., and HANKS, L. M. 2009a. Male-produced aggregation pheromone of the cerambycid beetle Rosalia funebris. J. Chem. Ecol. 35:96-103.

Ray, A. M., Swift, I. P., Moreira, J. A., Millar, J. G., and Hanks, L. M. 2009b. An aggregation pheromone produced by males of the desert cerambycid beetle Anelaphus inflaticollis (Coleoptera: Cerambycidae). Environ. Entomol. (in press).

Reddy, G.V.P., FettKöther, R., Noldt, U., and Detner, K. 2005. Capture of females Hylotrupes bajulus as influenced by trap type and pheromone blend. J. Chem. Ecol. 31:21692177.

SAS InSTITUTE. 2001. SAS/STAT User's Guide for Personal Computers, Release 8.01. SAS Institute, Cary, NC.

SOKAL, R. R., and RohlF, F. J. 1995. Biometry, 3rd ed. Freeman, New York, NY.

ZiLKOWSKi, B. W., and BARTELT, R. J. 1999. Cross-attraction of Carpophilus humeralis to pheromone components of other Carpophilis species. J. Chem. Ecol. 25:1759-1770. 